\title{
Oral Healthcare Measures to Improve Overall Health in Older Adults
} Uloma Onubogu' ${ }^{*}$, Ph.D., MSN, MSEd, APRN, W. Michael Mansfield², DDS, Irene N. Ozbek ${ }^{3}$, Ph.D

${ }^{1 *}$ School of Nursing, Florida A\&M University, 334 Palmer Avenue, Tallahassee, FL 32307, USA.

${ }^{2}$ School of Dentistry, University of Louisville, 501 South Preston Street, Louisville, KY 40202, USA.

${ }^{3}$ Psychology Department, University of Tennessee at Chattanooga, \#2803 540 McCallie Avenue, Chattanooga, TN 37403, USA.

Article Details

Article Type: Review Article

Received date: $01^{\text {st }}$ December, 2019

Accepted date: $19^{\text {th }}$ December, 2019

Published date: $21^{\text {th }}$ December, 2019

"Corresponding Author: Uloma Onubogu, School of Nursing, Florida A\&M University, 334 Palmer Avenue, Tallahassee, FL 32307, United States. E-mail: uloma.onubogu@,famu.edu

Citation: Onubogu U, Mansfield WM, Ozbek IN (2019) Oral Healthcare Measures to Improve Overall Health in Older Adults. J Comp Nurs Res Care 4: 156. doi: https://doi.org/10.33790/jenrc1100156.

Copyright: ( 2019 , This is an open-access article distributed under the terms of the Creative Commons Attribution License 4.0, which permits unrestricted use, distribution, and reproduction in any medium, provided the original author and source are credited.

\begin{abstract}
The purpose of this paper is to guide the understanding of preventive oral health measures to improve overall health in older adults. Oral health is a key indicator of overall health, well-being, and quality of life, yet, oral health status among older adults has remained alarmingly poor. Common oral conditions include dental decay, periodontal disease, tooth loss, dry mouth, and malodor. The connection between oral and systemic health has been established and this relationship is affected by various interrelated factors which pose many threats to older adults' health. Documented factors may range from chronic diseases, use of medications, individual lifestyle, decline in cognitive and physical functioning, access to care, to several social indicators of oral health. Dental disease and poor oral health are largely preventable through healthy lifestyle behaviors and effective management of chronic systemic diseases. A collaborative team approach is necessary to ensure that older adults receive comprehensive care appropriate for their complex medical and dental needs.
\end{abstract}

Key Words: Oral Health; Preventive Oral Health Measures; Older Adults; Oral and Systemic Connection; Periodontal Disease; Poor Oral Health, Social Determinants

The purpose of this paper is to guide the understanding of preventive oral health measures to improve overall health in older adults. Specifically, it is expected that readers will:

- Recognize the connection of oral health and systemic health.

- Describe the characteristics of poor oral health.

- Identify major risk factors associated with developing poor oral health.

- Describe effective preventive oral health care measures.

- Recognize common indications for immediate evaluation.

- Explain strategies to support caregivers in oral health care.

- Identify social determinants which impact oral health status.

- Discuss initiatives to improve access and outcomes in oral health.

\section{Overview}

Oral health means much more than healthy teeth [1]. According to the World Health Organization (WHO), oral health means the state of being free from chronic mouth and facial pain, oral and throat cancer, oral sores, birth defects such as cleft lip and palate, periodontal (gum) disease, tooth decay and tooth loss. Oral health also includes other diseases and disorders that affect the oral cavity and limit an individual's capacity in biting, chewing, smiling, speaking, and psychosocial wellbeing [2]. The comprehensive WHO definition of oral health encompasses dental health (pertaining to teeth and gum) which is often used synonymously with the term oral health.

The 2000 U. S. Surgeon General's Report [1] was the first to be devoted to oral health. Five key points of this report are that:

- Oral health means much more than healthy teeth.

- Oral health is integral to general health.

- Safe and effective disease prevention measures exist that everyone can adopt to improve oral health and prevent disease.

- There are profound disparities in the oral health of Americans.

- General health risk factors such as tobacco use and poor dietary practices affect oral and craniofacial health.

A survey of non-institutionalized US adults $(\mathrm{N}=1,636)$ aged 18 years and older indicated that the respondents considered oral health to be very important and equal in importance to certain medical conditions [3]. Oral health is recognized as a gateway to good health, but, despite improvements in oral health care and increasing awareness of its relationship to overall systemic health, oral health among United States (US) adults has remained alarmingly poor [4]. The poor rate of oral health is indicated by high prevalence of untreated caries, low rates of dental restorations, and high rates of edentulism (partial and complete loss of teeth) [5]. Millions of Americans are living with one or more oral diseases including cavities (dental caries), oral and pharyngeal cancers [6], and, for older adults, up to $70 \%$ prevalence of periodontal disease [7]. According to the Centers for Disease Control and Prevention (CDC) [4], over $30 \%$ of US adults have untreated tooth decay and one in seven persons aged 35-44 years has gum disease. Prevalence of gum disease worsens to one in four among older adults aged 65 years and older [8]. In the U.S., $20.5 \%$ of adults aged 65 and over have lost all of their natural teeth and more than 7,800 people, mostly older Americans, die from oral and pharyngeal cancers each year [4].

\section{Relationship of Oral Health to Systemic Health}

Oral health is a key indicator of overall health, wellbeing and quality of life [2,9]. The oral/systemic dynamic is affected by various interrelated factors in the older adult that pose a threat to good oral health and often impact overall health and quality of life [10]. These threats result generally from chronic diseases, poor nutrition, lack of oral health promotion, and low social support [11-13]. Beyond the threats, there is established evidence of a 
connection between oral health and chronic diseases such as diabetes, heart disease, stroke, respiratory/pulmonary conditions, rheumatoid arthritis, and osteoporosis [6,10,12,14-26]. Other factors detrimental to oral health include the use of certain medications (e.g., diuretics, antidepressants, and antihistamines) and lifestyle behaviors (e.g., smoking and drinking) [6,14,15,27]. For instance, there is widespread agreement that drugs and diseases are the principal causes of dry mouth (xerostomia) [28-30]. Medications from 42 drug categories and 56 sub-categories are considered to be inducers of dry mouth [31]. Complications of xerostomia include dental caries, candidiasis (thrush), and/or difficulty with the use of dentures [32]. As these conditions are prevalent among older adults, they impose a significant threat to oral health [5,9].

The American Dental Association [33] released the Action for Dental Health: Bringing Disease Prevention into Communities as part of its' comprehensive Dental Health Initiative. This initiative was designed to address the dental health crisis in three distinct areas:

1. Provide care now to people who are suffering from untreated disease.

2. Strengthen and expand the public/private safety net.

3. Disease prevention and dental health education.

Lack of access to oral health care is a major determinant to oral health. A variety of social, cultural, structural, and geographic factors have been found to hamper access to oral health care, thereby resulting in profound oral health disparities [34]. Major influences of access include gender, age, educational level, race and ethnicity, access to health insurance, and geographical location $[1,11,34,35]$. In fact, a major oral health goal of the Healthy People 2020 initiative [6] is to improve access to preventive services and dental care. In congruence, the CDC's current strategic plan (2011-2014) for oral health addresses public health priorities to eliminate disparities or inequalities in the receipt of health care services and health outcomes of different groups (ethnic, age, lifestyle, education, health behavior, income, insurance status, employment, housing, or geographical area) [36]. More recently, a new framework [37] advances a set of strategies and actions to resolve ongoing disparities in oral health under five overarching goals:

a) Integrate oral health and primary health care.

b) Prevent disease and promote oral health. c) Increase access to oral health care and eliminate disparities.

d) Increase the dissemination of oral health information and improve health literacy.

e) Advance oral health in public policy and research.

Many oral health problems are preventable among older adults. A position statement of the Academy of Nutrition and Dietetics [38] supports optimal systemic and topical fluoride as an important public health measure to promote oral and overall health throughout life. Simply, good manual dexterity and personal motivation are directly related to effective plaque removal and, therefore, impacts an older adult's ability to effectively clean their teeth. However, diminished cognition, decreased visual acuity or loss of strength in the hands may significantly alter a person's ability to maintain good oral hygiene [39]. Appreciating the ecology of the oral cavity is important. Tooth decay, like periodontal disease is now recognized as an infectious, transmissible, multifactorial disease of bacterial origin [40]. As well, dental plaque is a diverse community of microorganisms found on the tooth surface as a biofilm which is embedded in an extracellular matrix of host and microbial origins [41]. Thus, according to Marsh [42], the development of plaque-mediated disease (i.e. tooth decay or periodontal disease) at a site may be viewed as a breakdown of the homeostatic mechanisms that normally maintain a beneficial relationship between the resident oral microflora and the host.

Older adults in the U.S. are a dental caries active group. They have been found to experience new dental caries at a minimum rate as that of children ages $5-11(20.2 \%$ vs. $20.4 \%)$ and greater than that of the teenage population ages $12-19(20.2 \%$ vs. $13.3 \%)$ [5]. See Figure 1. As such, dentate older people should be targeted for intensive monitoring of dental caries and preventive efforts at both the clinical practice and public health levels [43]. The implementation of good oral hygiene protocols can significantly improve oral health and thereby greatly improve individuals' overall health. Simple and easy-to-initiate oral hygiene protocols include use of fluoridated drinking water and toothpaste, brushing at least twice daily utilizing a proper technique, flossing between the teeth at least once per day, proper care of dentures and supporting tissues (when applicable), and visiting the dentist for regular check-ups $[44,45]$.

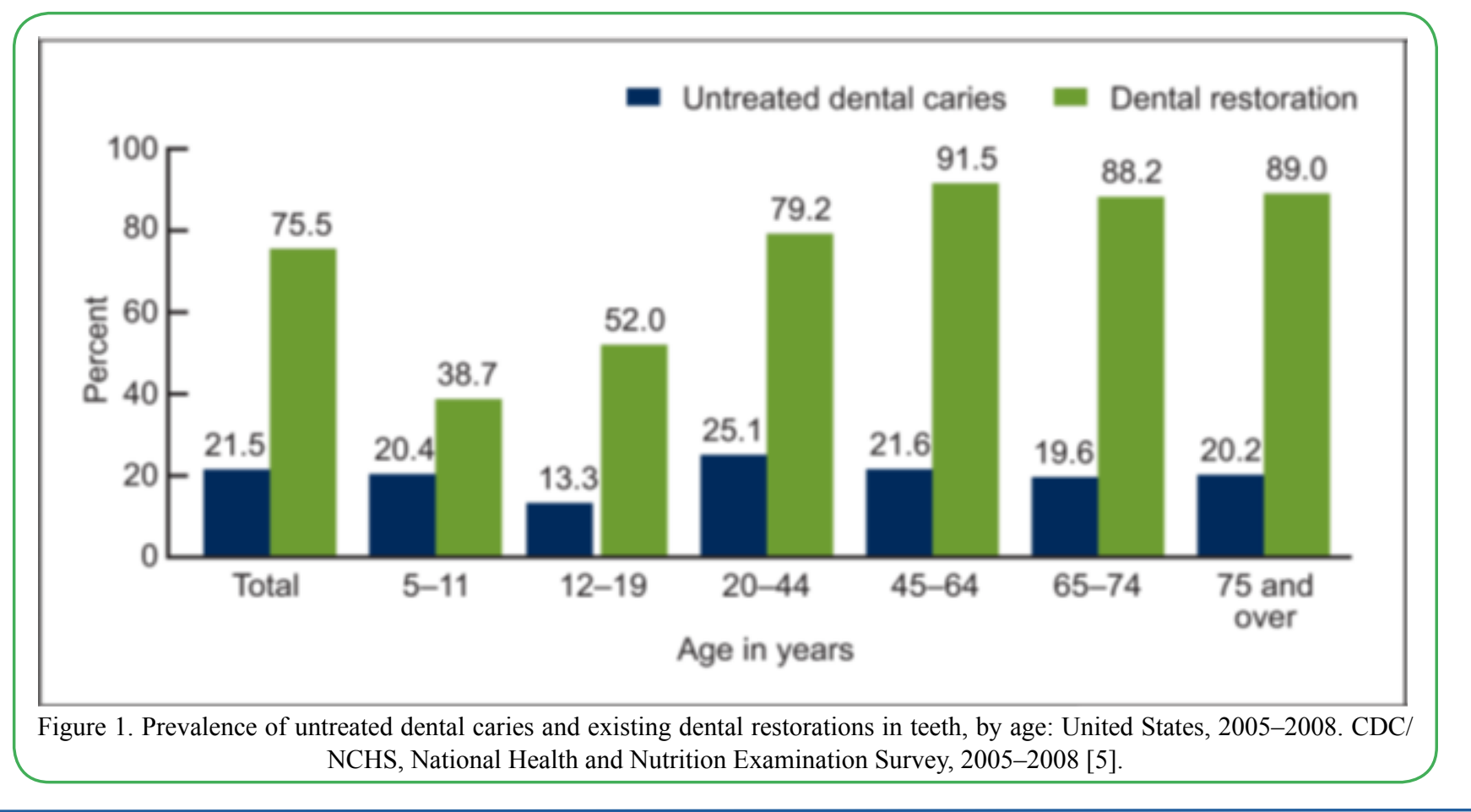

ISSN: $2581-3846$ 


\section{Components and Characteristics of Periodontal Disease and Poor Oral Health}

Question 1: Which of the following answers best describes periodontal (gum) disease?

a. The two components are Gingivitis -- the mildest form of gum disease, and Periodontitis -- an advanced state of gum disease.

b. It is a normal part of the aging process and there are no real effective measures a person can apply to address the problem.

c. It is a localized autoimmune condition with little if any systemic ramifications.

d. Approximately $30 \%$ of adult Americans over 65 are affected by periodontal disease ranging from gingivitis to periodontitis.

The correct answer is $A$, the two components are ... Gingivitis is a reversible condition, the mildest form of periodontal disease and is often caused by inadequate or poor oral hygiene [46]. Factors that may contribute to gingivitis include, diabetes, smoking, aging, genetic predisposition, systemic diseases and conditions, stress, inadequate nutrition, puberty, hormonal fluctuations, pregnancy, substance abuse, HIV infection, and certain medication use [4751]. If gingivitis is not diagnosed and treated early, it may progress to a more serious and irreversible condition called periodontitis. A person can return their gums to a healthy state without permanent damage with proper professional care, good oral hygiene practices, and diagnosing/treating periodontal disease at the gingivitis state before it progresses to periodontitis. In periodontitis, gums separate from the teeth, forming pockets (spaces between the teeth and gums) that become infected. As the disease progresses, the pockets deepen and more gum tissue and bone are destroyed. If untreated, teeth can eventually become loose and may have to be removed [46,52]. See Figure 2, periodontal disease produced by American Academy of Periodontology (AAP) [52].

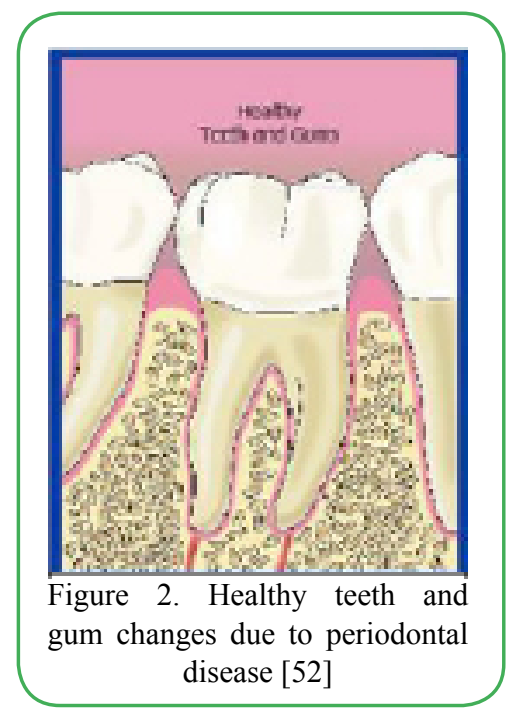

\section{Options B, C, and D are incorrect responses}

Periodontal disease should not be viewed as a normal part of aging. According to the AAP, periodontal disease is a chronic inflammatory disease of the gum tissue and bone supporting the teeth [52]. Utilizing good oral hygiene practices can help a person decrease the chances of getting periodontal disease. The American Dental Association (ADA) Mouth Healthy website identifies key components to a good oral care routine as brushing your teeth twice a day with an ADAapproved fluoride toothpaste, using dental floss or an interdental cleaner to clean between the teeth once a day, limiting snacks and eating a well-balanced diet, and visiting a dentist regularly for teeth cleaning and an oral exam [53].
The effect of good oral health on patient's overall health is an area of increasing research and interest [10]. A growing body of evidence suggests that maintenance of healthy teeth and gums is important not only to overall health of the mouth, but may be integral to overall health of the body and quality of life $[12,17,54]$. The proposition that an interaction exists between oral disease (i.e. periodontal disease) and systemic conditions such as diabetes, cardiovascular disease, aspiration pneumonia (bacterial), rheumatoid arthritis and stroke has been highlighted in numerous studies and investigations [12,16-20,22,55]. For example, having oral mucosal disease was associated with systemic inflammation marked by raised levels of c-reactive protein and increased prevalence of cardiovascular disease $[16,24]$. In other studies, improvement of oral health care diminished the risk of developing both aspiration and other acquired pneumonia and associated mortality $[20,21,56,57]$. Further understanding of the strength and nature of the relationship(s) between periodontal and systemic diseases should expand in the future with additional welldesigned observational studies and future random controlled trials [58]. According to the CDC's Oral Health Division [59], 47.2\% of adults aged 30 years and older and $70.1 \%$ of adults aged 65 years and older have periodontal disease. In addition, poor oral health is more common in men, those living below the poverty level, those with less than a high school education, and those individuals who currently smoke [59].

\section{Risk Factors for Poor Oral Health}

Question 2: Mr. Fey is an 80-year old resident of an Assisted Living Facility who suffers with dementia and diabetes. He is experiencing functional loss with activities of daily living. His primary caregiver is his 78-year old spouse who suffers with debilitating arthritis of the fingers in both hands. Both he and his wife are covered under the Medicare Part B program for their primary health care. Which of the following pairs of risk factors are recognized as priorities in planning oral health care for Mr. Fey?

a. Cognitive decline with selfcare deficits; Caregiver condition.

b. History of diabetes; Medicare coverage.

c. Current residence; Living with spouse.

d. Advanced age; Low literacy.

\section{The correct answer is $\mathrm{A}$, cognitive decline, self-care deficit}

Major risk factors for poor oral health include age, stroke, dementia, frailty, chronic diseases, and self-care deficit $[59,60]$. The immediate oral health care plan should recognize conditions that impact the individual's cognitive functioning and self-care ability, as these may result in the individual's inability to perform their own oral care [61]. A study by Park and colleagues [62] found that tooth loss was correlated with cognitive impairment among community dwelling adults aged 50 years and above, even without any medical history of stroke or dementia. Other studies found that increased risk of cognitive decline correlated with greater incidence/prevalence of tooth loss, periodontal disease, and caries [63]. This evidence shows that as the severity of impairment increases, the ability of individuals to perform self-care and basic activities of daily living such as hygiene and grooming decreases [64]. Frailty and functional decline increase the risk of poor oral health in older adults because of reduced ability to perform oral hygiene independently, and reliance on caregiver assistance. Reliance on caregivers may contribute to increased risk if the caregiver is physically unable to provide needed care or if they possess a poor understanding of the importance of oral care to overall wellbeing. Debilitating musculoskeletal disease, such as arthritis, can impede the ability of individuals to provide or assist with care. In this circumstance, Mr. Fey's spouse might find it difficult to handle care equipment efficiently because of her arthritis. Consequently, the neglect of oral care may result in serious consequences such as structural damage and tooth loss.

\section{Options B, C, and D are incorrect responses}

Although not considered as top priorities in the immediate care 
plan for Mr. Fey, current residency, history of diabetes, low literacy of patients or health care personnel, and Medicare non-coverage may significantly increase the risk of poor oral health. Residence in an Assisted Living Facility does not offer any guarantee for supervised regular oral health for the patient and may exacerbate patient's overall risk for poor oral health [65]. According to Griffin and colleagues [64], institutionalized and homebound adults who have self-care difficulty have poorer oral health than active elderly. According to this report, these vulnerable older adults confront barriers in accessing care mostly due to cost, lack of transportation, and limited mobility. Other factors attributed to poor oral health among institutionalized elderly are low information (literacy) and skill of healthcare personnel. Often, caregivers are unprepared to assume oral health ADLs and require training on strategies to overcome resistance to oral hygiene [13]. Oral health literacy, which is the degree to which a person is able to get, evaluate, understand, and use oral health information and services to make good decisions or act on them, involves more than just the ability to read $[37,66,67]$. An Institute of Medicine (IOM) [68] oral health literacy report identified concern about lack of awareness of basic risk factors and preventive regimens for many oral diseases among public and health care providers. This report associates limited oral health literacy to inaccurate knowledge about preventive measures. For example, low oral health literacy can impact patients' understanding of important oral health issues and information, eating practice, use of preventive services when available, and regular dental visits $[12,66,69]$. A lack of attention to oral health among primary care providers was also cited as one of the major factors associated with poor oral health [70]. Physicians, nurses, and other health care professionals have been reported as not adequately educated or trained in providing basic oral health care which includes recognizing oral diseases or teaching patients about selfcare [70]. A study of oral care provided by Certified Nursing Assistants in dentate long-term care residents found a generally deficient standard regarding skills, safety of performance, and knowledge [71]. Time spent providing oral care was not adequate and only $16 \%$ of residents who were dependent for oral care received it. Residents who had ability to perform oral care with supervision were frequently prompted or cued to stop. Frequently, oral care was provided under very poor hygienic and threatening conditions for the residents, usually with insufficient supplies to manage oral care. A study conducted to examine barriers to effective communication specific to older adults determined that literacy issues pertaining to both patients and providers were involved [72]. This research provided insight into verbal and non-verbal techniques to improve communication with older adults such as agenda preparation, listening, using simple language, and providing written instructions.

Advanced age by itself is not recognized as a factor for poor oral health. However, because of the high prevalence of chronic comorbidities, cognitive decline, and loss of capacity for self-care as individuals age, older adults may be at increased risk for poor oral health [61]. Individuals' overall health can be affected by their oral health and vice versa. A history of diabetes draws attention to the oral and systemic health connection $[6,10,14,15,24,27,61]$ and the need to mitigate risks through good diabetes control and good oral care. National Health and Nutrition Examination Survey (NHANES III) [73] provided evidence to support relationships among oral health status, chronic oral infections, and certain systemic diseases such as Type 2 Diabetes Mellitus (T2DM) and aspiration pneumonia. The NHANES III survey and other recent studies determined that oral health was an important etiologic factor in aspiration pneumonia $[20,21,25,73]$. As revealed in these studies $[19,74]$, there is sufficient evidence to support consideration of a bi-directional relationship between diabetes and periodontal disease as well as establish periodontal infection as a risk factor for poor glycemic control in patients with T2DM. $[19,73,74]$. Lamster's report went on to show that long standing and poorly controlled diabetes increased risk for oral candidiasis (oral thrush) [19]. In a recent study, poor dentition and lack of dental care were associated with worse healthrelated quality of life in older adults with DM [75]. For example, in conditions such as diabetes, the occurrence of candidiasis (oral thrush) has been attributed to elevated blood sugar levels, and the report of permanent tooth loss due to caries or periodontal disease was more likely in patients with DM than those without [76].

Older adults generally experience difficulty getting effective preventive oral care due to lack of health insurance compared to younger people [64]. In the United States, the majority of older adults are covered under the Medicare program (Parts A \& B) for their health care [77]. Medicare Part A covers healthcare involving hospitalization, while Medicare Part B covers for primary health care services in various settings including ambulatory, long term, and home health care [77]. Dental service under the Medicare program is limited, covering only services that are termed medically necessary $[77,78]$. The covered services include x-rays performed prior to reduction of jaw fracture, tooth extraction performed in preparation for jaw radiation treatment, or oral examination performed prior to kidney transplant or heart valve replacement. Services are covered by Medicare Part A if performed by a dentist on the hospital staff or by Medicare Part B if performed by a physician. These stipulations exclude from coverage routine dental care (oral exams, checkups, screening, and cleanings) and other primary dental service for the care, treatment, filling, removal, or replacement of teeth or teeth structures [77,79]. Thus, important services such as preparation of the mouth for dentures, and removal of diseased teeth in an infected jaw are impacted. Without an alternative dental plan, access to professional treatment is impeded for many. Sources through which some Medicare beneficiaries obtain dental coverage include Medicare advantage, Medicaid, employer-sponsored retiree health plans, or individually purchased plans [79]. An employer-based plan usually covers dental care, and Medicare Advantage covers specified number of exams, cleanings, or x-rays, per year, but, not restorative dental services, such as fillings, crowns, or dentures. Because employerbased dental plans are associated with employment, many older adults loose this benefit when they retire $[64,80,81]$. Thus, many older Americans still remain without insurance coverage for dental care $[79,80,82]$. It has been reported that as many as $70 \%$ of older adults are without any form of dental insurance $[64,65]$. The high rate of uninsured or under-insured was attributed to high cost of coverage through private dental insurance and poor dental coverage for low income adults through the Medicaid program which is challenged by limited service, poor provider reimbursement, and shortage of providers [82-84]. Considering that only $22 \%$ of older persons are covered by dental insurance (private or employer-based), while most pay out-of-pocket for their dental expenses $[81,85]$, it is clear that lack of dental insurance for older Americans is a major barrier to dental care [82].

\section{Preventive Oral Healthcare Measures}

Question 3: Mr. Fey has retained majority of his natural teeth and he does not wear dentures. Which of the following measures will not help Mr. Fey and his caregiver to decrease his risk for developing dental or periodontal disease?

a. Daily fluoride rinse.

b. Tooth whitening agent.

c. Flossing twice daily.

d. Regular dental visit.

e. Adequate hydration.

\section{The correct answer is $B$, tooth whitening agent}

Tooth whitening (bleaching) does not contribute to decreased risk for periodontal disease. It is a cosmetic procedure and not a therapeutic agent.

Options A, C, D, and $\mathrm{E}$ are incorrect responses

According to the CDC [86], water fluoridation is recognized as 
one of 10 great public health achievements in the 20th century. Appropriate use of fluoride has many beneficial effects on overall oral health which can be cumulative. For example, fluoridation reduces tooth decay by $25 \%$ over a person's lifetime [86]. Accordingly, people who drink non-fluoridated bottled water or live in rural or urban areas without community fluoridated water (CFW) sources may be at increased risk for dental decay [86]. Fluoridated mouth rinses and fluoride toothpaste have been shown to provide additional tooth decay reducing and reversing benefit daily [87,88]. Equally, flossing daily is a good preventive protocol for oral health. Flossing cleans between the teeth where a tooth brush cannot reach $[87,88]$. Additionally, cleaning the tongue and edentulous gum tissues is an important preventive oral care practice. A caregiver's ability to assist with oral care can be challenged by conditions that interfere with manual dexterity such as arthritic fingers. Thus, handling of oral care tools such as a dental floss might be potentially difficult for Mr. Fey's caregiver with results that might impact the overall state of his oral health. A variety of assistive aids such as floss holders and floss threaders can make it easier for the arthritic person to use floss. Regular visits to the dentist are an important part of oral health. In addition to checking for cavities or tooth decay and checking for gum disease, the exam evaluates the effectiveness of brushing and flossing by the patient and/or caregiver. Frequently, older adults are on a variety of medications that can cause dry mouth as a side effect thereby reducing the protective production and function of saliva $[30,89]$. For these patients, interventions to relieve dryness and prevent adverse consequences may include frequent brushing with fluoride toothpaste or rinse, frequent oral fluid intake, using sugarless gums containing xylitol, avoiding tobacco and alcohol, and using artificial saliva preparations if needed for additional relief [90,91].

Question 4: During a recent dental visit, Mrs. Leary - a 67 year-old retired African American - learned that she has advanced periodontal disease, tooth caries involving two teeth, and a piece broken from a molar which she does not recall happening. She claims that she brushes every morning and does not understand the poor state of her oral health. Mrs. Leary states that she feels she is in good general health. However, she has a 10-year history of Type 2 Diabetes and chronic tobacco use of half a pack of cigarettes a day. She has a healthy appetite and consumes 3 glasses of white wine sipped slowly over lunch and dinner every day. Her nightly routines include a cup of warm chocolate milk in bed as she slowly falls asleep. She has not received dental care in the last two years since retirement because she says that her social security income doesn't stretch far enough to cover visits to the dentist. In providing oral health education to Mrs. Leary, which of the following will be identified as preventable lifestyle risk factor for poor oral health?

a. Alcohol abuse, cost of dental care, and professional treatment.

b. Cost of dental care, dental caries; broken molar.

c. Diabetes, daily tooth brushing, dental caries.

d. Poor oral care practice, smoking, and excessive alcohol use.

The correct answer is $D$, poor oral care practice, smoking, and excessive alcohol use

Dental disease is largely preventable with healthy lifestyle behaviors. However, many older adults have poor oral health due in large part to unhealthy lifestyle behaviors, including tobacco use, excessive alcohol use, and poor dietary choices $[1,92,93]$. Consumption of more than one drink of any wine (red or white) per day by Mrs. Leary is considered excessive in women as this exceeds 8 drinks per week [94]. See Figure 3 [94]. CDC also considers excessive alcohol use a high risk behavior for developing oral cancers $[62,94]$. Another major and growing concern is the destruction and loss of tooth from acid erosion (higher acidity is present in white wine) associated with excessive alcohol consumption [95]. This is especially relevant if excessive use of alcohol is accompanied by poor oral hygiene, a common behavior in persons with alcohol abuse problem. Inappropriate use of wine can lead to extensive dental erosion [95]. Wine (white, red, sweet) mixed with saliva through vigorous wine rinsing was found to significantly reduce intra-oral $\mathrm{pH}$ to $<5.5$ within 15 seconds and lasting up to 45 seconds, thereby increasing the risk of developing dental erosion [96]. In a similar report, Mandel [95] called attention to wine acidity $(\mathrm{pH}=3.0$ to 3.8) which far exceeds the acidity at which enamel dissolves $(\mathrm{pH}$ $=5.0$ to 5.7) in order to alert patients and dentists of its role in oral health. Manarte and colleagues [97] found a high occurrence of dentine erosion (> 49\%) among alcoholic patients (age 42-67 years) undergoing detoxification. Eating or drinking before bed without proper oral care is a lifestyle that increases the risk for dental decay from bacterial and chemical activity. Dental erosion can begin as soon as 15 to 61 seconds of holding wine in the mouth as when a person sips slowly over a prolonged period [95]. Mrs. Leary faces potentially higher risk for dental erosion because of higher acidity in white wine, compared to red wine $[95,98]$. Reduction or avoidance of alcohol and tobacco use will greatly improve overall risk for adverse oral health outcomes. Good self-care practices such as twice daily tooth brushing with fluoride toothpaste, and daily flossing are positive behaviors for promoting good oral health $[1,86,92,93]$. These practices are equally beneficial after food or drink and before bedtime. However, it has been shown that these behaviors decline with age [64]. Therefore, routine assessment and promotion of healthy oral health care practices are essential in the care of older adults.

\section{Options A, B, and C are incorrect responses}

The cost of dental care, dental caries, and a broken molar are not lifestyle behaviors. Dental caries and broken molars are common outcomes of alcohol abuse and poor oral health $[60,64]$. High cost of dental care from out of pocket spending is a barrier that has limited the use of preventive interventions and treatments for many older adults [86,99-101]. According to AARP report [99], older adults incur their largest out of pocket spending (42\%) on non- covered dental care expenses on the Medicare insurance program [99]. For more than half of older adults living on fixed or low annual income of less than $\$ 22,000$, lack of insurance and high personal cost constitutes barriers to seeking needed dental care [79]. The US Department of Health and Human Services (USDHHS) [1,92] identifies other barriers as limited access to and availability of dental services, lack of awareness of the need for care, and fear of dental procedures.

\section{Symptoms Requiring Immediate Evaluation by a Dentist}

Question 5: Which of the following oral symptoms is not a reason to contact a Dentist as soon as possible?

a. Sores in the mouth.

b. Pain and bleeding in the mouth area.

c. Stained teeth.

d. Swelling in the mouth.

e. Persistent odor of bad breath.

\section{The correct answer is $\mathbf{C}$, stained teeth}

Emergency situations require immediate assessment by a dentist. While a stained tooth or teeth may be esthetically unpleasing, it is not typically an emergency situation. Stains have been traditionally classified as either Intrinsic or Extrinsic. [102,103]. Intrinsic stains are those stains incorporated within the tooth structure that cannot typically be removed by tooth brushing or teeth cleaning at a dental office. A number of metabolic diseases and systemic factors are known to affect teeth during fetal development and cause discoloration or staining [102]. Examples are listed in Table 1 [102]. Causes of intrinsic stain include dental fluorosis (which typically appears as white-spotted-brown pitted enamel), tetracycline stain (which typically appears as grayish-brown discoloration), hypocalcification (white spots on enamel), or demineralization (white or brown spots on enamel) [103]. Extrinsic stains occur on the tooth and can usually be removed by tooth brushing or teeth cleaning at a dental office. Causes of this type of stain include color-producing bacteria, use of staining substances such as wine, coffee, tea, soft drinks, pigmented 
berries, some drugs and exposure to metallic compounds. The color of stain produced depends on the agent causing the stain [103]. A third category called internalized stain or discoloration is sometimes used to describe extrinsic stains which are also incorporated within the structure of the tooth after development $[102,103,104]$. An example of this type of stain is the discoloration of a tooth that occurs after root canal therapy. A recent study found that all endodontic materials cause measurable color change [105].

\section{Alcohol Use and Your Health}

\section{Drinking too much can harm your health. Excessive alcohol use leads to about 88,000 deaths in the United \\ States each year, and shortens the life of those who die by almost 30 years. Further, excessive drinking cost the economy $\$ 249$ billion in 2010. Most excessive drinkers are not alcohol dependent.}

\section{What is considered a "drink"? U.S. Standard Drink Sizes}

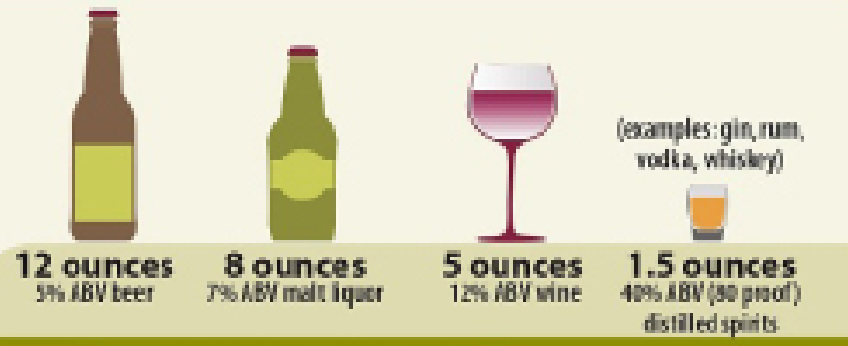

\section{Excessive alcohol use includes:}

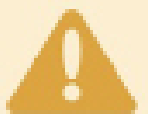

Binge Drinking

fer wamen, 4 or nene dinke consamed on one ocrasion
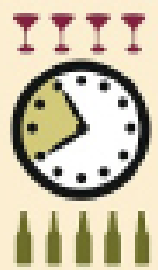

For nen, 5 ar more trinks conaland on one occasion

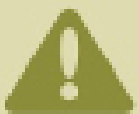

Heavy Drinking

for wamn, 8 or mane dineks

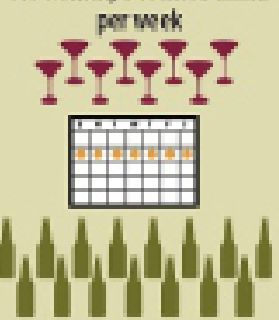

for nen, 15 crmore dinks per week

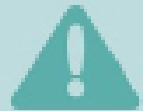

Any alcohol used by pregnant women

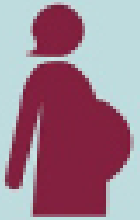

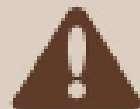

Any alcohol used by those under the age of 21 years

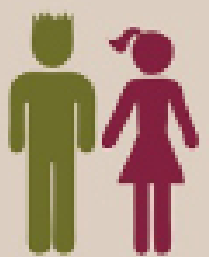

\section{If you choose to drink, do so in moderation:}

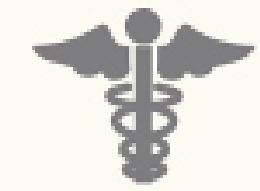

LONT DENS A ALL ifyou are under the age of $21, x$ if yware ar may bepeguant. or have health problens that could temade worse by erinking.

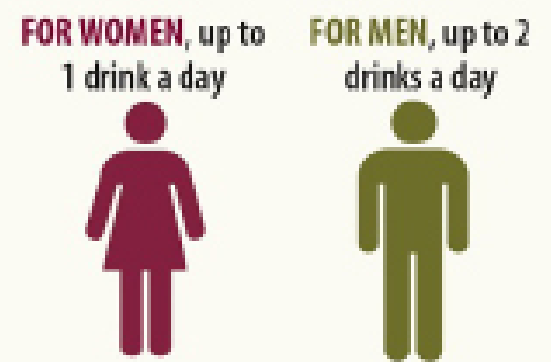

KO ONE Should begin dfitking or dinkmarefrequently based on potential heslth benefits. 
Intrinsic Stains

Dental Fluorosis

Tetracycline stain

Hypocalcification

Demineralization

Extrinsic Stains

Internalized stain or discoloration
Stains incorporated within the tooth structure that cannot typically be removed by tooth brushing or teeth cleaning at a dental office.

Excessive fluoride ingestion during enamel development

Excessive tetracycline ingestion during enamel development

Frequently results from high fever "white spots" on enamel during enamel formation

Acid erosion of enamel caused by plaque and plaque byproducts

Stains which occur on the tooth and can usually be removed by tooth brushing or teeth cleaning at a dental office. Causes include color-producing bacteria, use of staining substances such as wine, coffee, tea, soft drinks, pigmented berries, some drugs and exposure to metallic compounds

Extrinsic stains which are incorporated within the structure of the tooth after development. For example, the discoloration of a tooth that occurs after root canal therapy or bleaching of a non-vital tooth

Table 1. Classification of stains developed by the authors using information from Watts \& Addy [102]

\section{Options A, B, D, and E are incorrect responses}

Occurrence of these oral symptoms or conditions will require immediate evaluation by a dentist:

Sores in the mouth: A sore in the mouth is a valid reason to contact a Dentist. Any white or red lesion that persists for longer than two weeks should be referred to a Dentist or dental specialist (dental specialist such as an oral surgeon or oral pathologist) for evaluation [39]. There are a variety of conditions that may cause a sore(s) in the mouth, as well as different types of mouth sores. Not all sores or ulcers are cancerous or infectious in nature. Traumatic ulcers may be caused by etiologic agents such as hot food, cheek biting, tongue biting in addition to actual trauma to the mouth. The American Dental Association [106] lists a number of mouth sores including:

- Canker Sores: These sores are typically small, white or gray, have a red border.

- Cold Sores or Fever Blisters: These sores are caused by the Herpes Simplex Virus and typically appear around the lips, under the nose or on the chin as clusters of raised blisters which can break and then form a crusty scab. They are contagious.

- Leukoplakia: These sores are usually white in color, not painful or contagious and typically arise from an irritation such as smokeless tobacco use or an ill-fitting denture. Note that Leukoplakias may be associated with oral cancer and therefore should be referred to a Dentist for appropriate diagnosis or referral. Some oral cancers are red in color (erythroplakias) or may be mixed (red and white) in color.

- Oral Thrush: This condition is often called oral candidiasis and is caused by a fungus - candida albicans. The sore is typically a white patch with a red base that may be wiped off but may bleed as a result. This condition is often present in immunosuppressed individuals and may be caused by some steroids, antibiotics or cancer therapies. In the erythematous clinical classification of candidiasis, the dorsal aspect of the tongue and the palate are generally involved and the patient may appear with painful, red mucosa with loss of papillae on the tongue and patchy red changes on the palate $[107,108]$. In older adults who wear dentures, colonization of the denture surface and underlying oral mucosa with oral candidiasis species may result in red inflamed (erythematous) and edematous tissue (denture stomatitis) in the denture-bearing areas $[39,109]$. Risk factors for denture stomatitis include poor denture fit and poor dental hygiene.

Pain and bleeding of the gums: Pain in the mouth may be divided into soft tissue pain and tooth pain. Bleeding gums may often be associated with poor oral hygiene and inflammation of the gums associated with periodontal disease (gingivitis and/or periodontitis [110,111]. Poor oral hygiene over time can lead to the development of plaque which is a biofilm composed of bacteria and toxins. Plaque develops on the teeth at the edge or margin of the gums. This leads to inflammation of the gums or gingivitis which is characterized by red and swollen gum tissue which can bleed easily on brushing [39]. Gingivitis is more common among older persons than younger, but age alone is not a risk factor for gingivitis or periodontitis [112]. Left untreated, gingivitis can progress to periodontitis. As periodontitis progresses, bacterial toxins and byproducts of the body's natural defense mechanisms start to break down the bone and connective tissue that support the teeth. Over time, there is swelling of the gums that leads to the formation of periodontal pockets which are difficult for a person to keep clean. The result can be increasing mobility of the tooth and eventual tooth loss. Both gingivitis and periodontitis may be painful conditions [113]. Other causes of gingival bleeding include bleeding disorders, brushing teeth too hard, hormonal changes, idiopathic thrombocytopenic purpura, poorly-fitting dentures, improper flossing technique, tooth or gum related infection, leukemia, use of blood thinners, and vitamin K deficiency [114].

Swellings and bumps in the mouth: There are swellings that can occur in the mouth, are acute and may necessitate that a Dentist be called as soon as possible. One classification of lesions which may 
present as swellings or bumps in the mouth divides the lesions into the following categories: normal, developmental, inflammatory, traumatic, cystic, hormonal, drugs, blood dyscrasias, neoplasms, and others [115]. Inflammation from an abscess is one example of an inflammatory lesion. There are normal oral anatomical structures which the caregiver may easily mistake as pathological, such as the orifices of the parotid glands. These raised areas of soft tissue are located on the cheeks next to the upper maxillary second molars. There are examples of hard (bony) tissue areas in the mouth which may also be mistaken for pathological lesions by caregivers such as palatal and mandibular tori. A palatal torus is frequently found in the midline of the hard palate and, while not present in every person, is not pathological. Mandibular tori may or may not be bilaterally present but are typically located on the inside of the mandible near the canine and premolar tooth region [116]. Unless a torus or tori make(s) eating or making of a prosthesis (partial or complete denture) difficult, they typically do not require surgical removal but should be noted, observed, and verified by a Dentist.

Bad breath or Halitosis: The prevalence of bad breath or halitosis (oral malodor) in the U.S. has been estimated at between 25 million and 85 million Americans depending on the source of the data [117]. Depending on the population studied, up to $50 \%$ of the population may have (or perceive they have) bad breath, and, for up to $50 \%$ of these people, the problem can impair the quality of life creating personal discomfort and social embarrassment [118-121]. Bad breath may have extrinsic and/or intrinsic causes. The most likely extrinsic cause of bad breath is the accumulation of food debris and plaque on the teeth and tongue, resulting from poor oral hygiene and resultant inflammation of the periodontal tissues (gingivitis and /or periodontitis). Other extrinsic causes include use of tobacco, certain foods, and certain spices. Extrinsic causes of bad breath are best removed by eliminating the intake of causative substances. Intrinsic causes of bad breath may be oral or systemic in origin. Approximately $90 \%$ of these cases are of intraoral origin and $10 \%$ are of systemic origin [122]. Oral causes of malodor or bad breath include tongue biofilm coating (due to poor oral hygiene), plaque-related gingival and periodontal disease, ulceration due to malignancy, medication related hyposalivation, candidiasis from wearing dental appliances, dental abscesses, and bone conditions such as dry socket [123]. However, acute necrotizing ulcerative gingivitis (trench mouth) causes the most notable bad breath. Systemic (extra-oral) causes of halitosis include respiratory, gastrointestinal tract, and metabolic disorders [118]. Because chronic or acute bad breath may be a sign of periodontal disease or a systemic condition, a dentist should be contacted if the symptoms are not extrinsic and do not resolve upon removal of the given agent (i.e., garlic). With the high prevalence of xerostomia, poor oral hygiene and chronic conditions among older adults, it can be postulated that bad breath occurs at an even greater frequency among older compared with younger adults.

\section{Strategies to Support Caregivers in Oral Health Care}

Question 6: Mr. Tee is living in a nursing home and is dependent for functional care due to dementia. When he was handed a toothbrush with toothpaste applied, he did not proceed to put the toothbrush in his mouth. When the caregiver guided his hand toward his mouth, he became resistant turning his head back and forth. Resistance to care was strong. Training for caregivers on what the next 'best step' might be would focus on teaching caregivers to:

a. Document Mr. Tee's choice when he refused care. Documentation would include a detailed description of Mr. Tee's behavior and the entire series of events so others can understand why his teeth may have plaque building up over time.

b. Use learning principles, i.e. stay at eye level with Mr. Tee, speak using nouns and gestures, smile, demonstrate the act of brushing teeth, use physical prompts gently and repeat the sequence until he is brushing his teeth. c. Ask for a medication consult regarding the addition of a sedative/ anti-anxiety medication to decrease Mr. Tee's resistance to care and make the task of dental care for the caregiver easier.

d. Talk to Mr. Tee as you would a child about the necessity of good dental care and brushing, making the use of a toothbrush fun, pretending it is an 'airplane that needs to land' for example.

\section{The correct answer is $B$, use learning principles}

An intervention, in which a pleasant tone of voice with physical gestures demonstrating teeth being brushed coupled with a gentle presentation of a toothbrush over and over until cooperation is elicited, has been shown to reduce care resistant behavior (CRB) [124]. Brushing teeth in a bathroom provides cues for brushing of teeth. Having Mr. Tee look in a mirror with the caregiver standing behind him demonstrating the brushing of teeth also provides cues for brushing of teeth [124]. A toothbrush paired with a pleasant voice in repeated learning trials allows the once-feared object to be associated with smiles and reassurances [125]. Reinforcement follows when Mr. Tee is thanked profusely for helping by opening his mouth. The clean taste that remains following brushing of teeth is rewarding also. A small amount of toothpaste on a soft toothbrush is sufficient to achieve result. If Mr. Tee has severe problems swallowing, special toothettes and brushes with suctions are available. Although the use of toothettes is not an adequate substitution for brushing teeth, as they are not effective in removing plaque, they can be useful in improving the appearance of oral tissues when patients are intubated or nonresponsive [126,127]. See Figure 3.

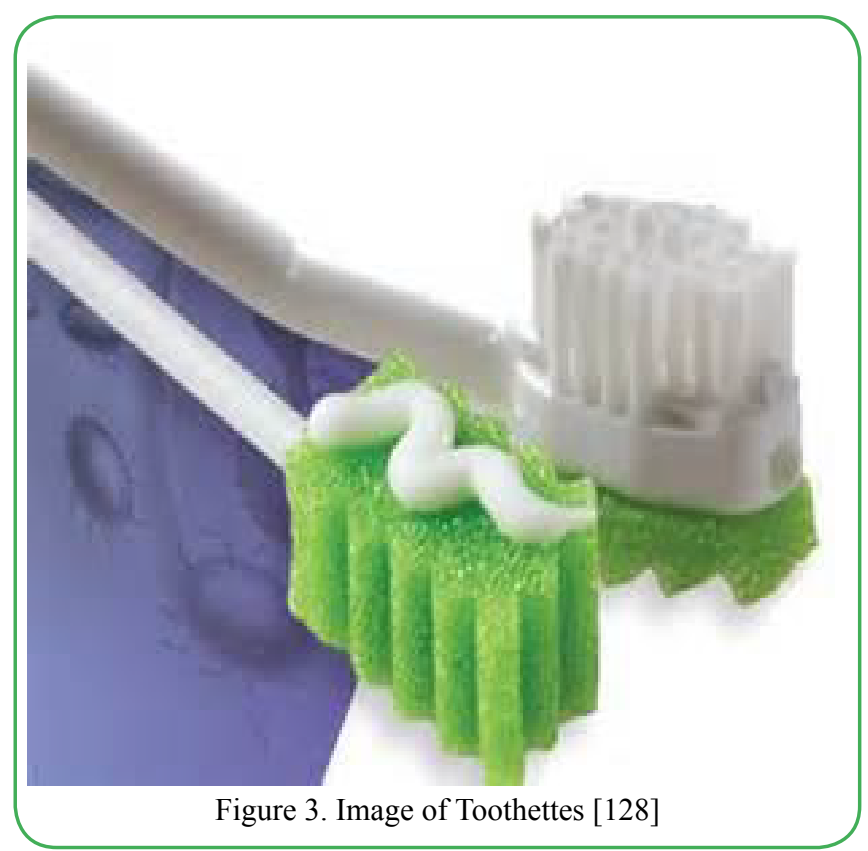

\section{Options $A, C$ and $D$ are incorrect responses}

Documentation does help communicate concerns to others, but documentation alone does not directly address the problem of Mr. Tee initially refusing care. Although Mr. Tee's initial refusal to allow the caregiver to assist in guiding the toothbrush toward his mouth may reflect his fear or his lack of understanding of what is happening, anti-anxiety medication does not solve this problem. Inappropriate use of medication may result in adverse outcomes for the patient. Mr. Tee's refusal to participate in care may seem similar to resistive behavior observed in young children. However, Mr. Tee is not a child and talking to him as one is not appropriate. When 33 residents living in long term care facilities were interviewed about their perceptions of how staff communicated with them, they reported unwelcomed ageism in the staff's use of language [129]. Elderspeak, the child like speech used when talking to older adults, is a known trigger for CRB [130]. 


\section{Social Determinants in Oral Health Promotion}

Question 7: Individual lifestyle behaviors interact with environmental factors to influence the quality of oral health. Which of the following socio-behavioral factors will not be a primary focus in oral health promotion?

a. Living condition.

b. Dietary/food choices.

c. Use of tobacco and alcohol.

d. Oral hygiene.

e. Exercise habits.

\section{The correct answer is $\mathbf{E}$, exercise habits}

There is no evidence linking exercise habits to oral health. However, several social determinants of health involving socio-economic and socio-behavioral factors do affect oral health [62]. Socio-economic factors include low socioeconomic status, poor living conditions, and ability to cope with stress $[93,131,132]$. Socio-behavioral factors include unhealthy food choices, use of tobacco and alcohol, and poor oral hygiene [84,29]. In general, disparities (oral health gap) exist in dentition and oral health status of US adults. People with lower levels of education and lower family income, and people from specific racial/ ethnic groups (non-Hispanic black and MexicanAmericans), have higher rates of disease, lower restoration rates, and more missing teeth [133]. A high prevalence of unmet dental needs ranging from tooth loss, pain, difficulty eating and chewing, was found among seniors (older adults). Among older adults aged 65 years and older, racial/ethnic minority was related to higher rate of untreated dental disease, more tooth loss, and lower oral healthrelated quality of life [64].

Oral health problems such as dry mouth and tooth loss contribute to poor dietary intake. Older adults with severe tooth loss are less likely than those with moderate to low tooth loss to meet current dietary recommendations [134]. Food avoidance and modification due to oral health problems are associated with significant differences in dietary quality [135]. In one study, older adults reported selectively avoiding foods such as whole grains, and fruits and vegetables, due to severe dry mouth or severe tooth loss [134-136].

\section{Improving Access and Outcomes in Oral Health Care}

Question 8: Federal initiatives to promote oral health in the US include Healthy People 2020, which targets the achievement of several important outcomes for US adults. Dentists and dental health care professionals can play a central role to reduce adverse outcomes in oral health. Appropriate outcome foci to impact oral health care include:

a. Increase awareness of the importance of oral health.

b. Reduce disparities in access to oral health care.

c. Increase referrals for diabetes testing.

d. Reduce the number of older adults who have lost all their teeth.

e. Increase acceptance and adoption of preventive programs.

f. A, B, D, and E.

g. All of the above.

\section{Answer G, All of the above, is correct}

Oral health is a leading health indicator in the US and plays a significant role in the overall well-being of individuals across the lifespan. Access to oral health care must be recognized as a priority in the healthcare system because many Americans of all ages do not have access to dental care despite significant improvements in overall oral health status in the last 50 years $[6,137]$. Up to $16 \%$ of seniors overall as well as higher numbers of non-Hispanic black and Hispanic seniors, and those with lower income and education report poor conditions of their teeth and mouth [138]. To address issues of poor access to dental care, recommended strategies are those that impact health behavior, reduce tooth decay, increase monitoring of oral diseases, increase the capacity of US states to provide preventive oral health services, and increase the oral health component in community health centers [6]. Implementation of these strategies will increase acceptance and adoption of preventive oral health programs in more communities nationally, with consequently fewer adverse outcomes such as tooth loss. Dentists and dental hygienists will play central roles in progress toward achievement of these goals, by providing education and counseling on reducing tobacco use and smoking cessation, by increasing the number of adults who receive screening for oral and pharyngeal cancer, and by increasing referrals for diabetes testing or management [6]. In conjunction with these national strategies, a recent Health Resources and Services Administration (HRSA) initiative is directed at integrating oral health in primary health care - a bold and welcome undertaking to increase access to oral health care $[33,34,139]$. To begin this integration, HRSA [139] has provided five domains of focus containing core clinical competencies for primary care practices. These domains include risk assessment, oral health evaluation, preventive interventions, communication and education, and inter-professional collaborative practice. The goal is that with additional training, interdisciplinary primary care teams of physicians, nurses, physician assistants, and others will be able to make primary oral health care more accessible and affordable to vulnerable groups such as older adults.

\section{Conclusion}

Good oral health is key to good overall health and essential to healthy aging and quality of life. A person's ability to speak, smile, smell, taste, chew, swallow, and make facial expressions are all improved with and connected to good oral health. Oral health is associated with a number of systemic conditions, and increasing the understanding of the oral-systemic health connection among all healthcare providers is important. A lack of access to oral health care contributes to significant oral health disparities in the US. Factors hampering access to oral care include a variety of social, cultural, economic, structural, and geographic variables. The cost of maintaining good oral hygiene and access to preventive services are minimal compared to the high cost of restorative care which can become necessary as a result of neglect and/or poor oral care. Thus, oral health care of older adults requires a team approach to ensure that complex medical and dental needs receive appropriate and comprehensive care. This will also involve efforts to address specific behavioral, socio-economic, and regulatory barriers to oral health care access, and increase the awareness of the importance of oral health in all patients, particularly older adults.

Conflict of interest: The authors have declared no conflict of interest.

\section{References}

1. US Department of Health and Human Services (2000) Oral Health in America: A Report of the Surgeon General-- Executive Summary. Rockville, MD: US Department of Health and Human Services, National Institute of Dental and Craniofacial Research, National Institutes of Health.

2. World Health Organization. Oral health. September 2018.

3. Carr A, Beebe T, Jenkins S (2009) An assessment of oral health importance. J Am Dental Associa140: 580-586.

4. Center for Disease Control (2011) Oral health; preventing cavities, gum disease, tooth loss, and oral cancers: at a glance.

5. Dye BA, Li X, Beltrán-Aguilar E (2012) Selected oral health indicators in the United States, 2005-2008.

6. U.S. Department of Health and Human Services (2020) Office of Disease Prevention and Health Promotion. Healthy People 2020. Topics and objectives. Oral health.

7. Eke PI, Dye BA, Wei L (2015) Updates on prevalence of periodontitis in adults in the United States. NHANES 20092012. J Periodontol 86: 611-622.

8. Center for Disease Control (2009) New series of reports to monitor health of older Americans. Oral Health. National Center for Health Statistics. 
9. Mertz EA (2016) Dental-Medical divide. Health Affairs (Millwood) 35: 2168-75.

10. Slavkin HC, Santa Fe Group (2017) A National imperative. Oral health services in Medicare. J Am Dental Associa 148:281-283.

11. Metcalf S, Northridge M, Lamster I (2011) A systems perspective for dental health in older adults. American Journal of Public Health 20: 1820-1823.

12. Atchison KA, Glicken AD, Haber J (2019) Developing an interprofessional oral health education system that meets the needs of older adults. J California Dental Associa 47: 247-254.

13. Chavez EM (2019) Older adults: the long and winding road to address their needs. J California Dental Associa 47: 213-214.

14. American Academy of Periodontology (2019) Periodontal disease and systemic health.

15. Malkin,B(2009)Theimportanceofpatients'oralhealthandnurses' role in assessing and maintaining it. Nursing Times 105: 19-23.

16. Federle S, Sabbah W, Donos N, Porter S, D'Aiuto F et al. (2011) Common oral mucosa diseases and systemic inflammation and cardiovascular diseases in a large cross-sectional survey. Am Heart J 161: 344-50.

17. Linden GJ, Herzberg MC (2013) Periodontitis and systemic diseases: a record of discussions of working group 4 of the Joint EFP/AAP Workshop on periodontitis and systemic diseases. J Periodontol 14: S20-S23.

18. Mealey, B (2006) Periodontal disease and diabetes. A two-way street. J Am Dental Associa 137: 26S-31S.

19. Lamster I, Lalla E, Borgnakke W, Taylor G (2008) The relationship between oral health and diabetes mellitus. J Am Dental Associa 139: 9S-24S.

20. Tada A, Miura H (2012) Prevention of aspiration pneumonia (AP) with oral care. Archives Gerontol Geriatr 55: 16-21.

21. van der Maarel-Wierink CD, Vanobbergen JN, Bronkhorst EM, Schols, JM, de Baat C (2013) Oral health care and aspiration pneumonia in frail older people: a systematic review. Gerontol 30: 3-9.

22. Maresz K, Hellvard A, Sroka A, Adamowicz K, Bielecka E, et al. (2013) Porphyromonas gingivalis Facilitates the Development and Progression of Destructive Arthritis through Its' Unique Bacterial Deiminase (PAD). PLOS Pathogens 9: 1-10.

23. Center for Oral Health (2018) A healthy smile never gets old: a California report on the oral health of older adults.

24. Taylor GW, Borgnakke W (2008) Periodontal disease: associations with diabetes, glycemic control and complications. Oral Disease 14: 191-203.

25. Gil-Montoya JA, Subira C, Ramon JM, Gonzalez-Moles MA (2008) Oral health-related quality of life and nutritional status. Journal of Public Health Dentistry 68: 88-93.

26. Madianos PN, Koromantzos PA (2018) An update of the evidence on the potential impact of periodontal therapy on diabetes outcomes. J Clini Periodontol 45: 188-195.

27. Kumar J, Dash R (2019) A conceptual framework for improving oral health among older adults: application of the spectrum of prevention strategies. J California Dental Associa 47: 257-263.

28. Sreebny L, Vissink A (2010) Dry Mouth, The Malevolent Symptom: A Clinical Guide.

29. Braverman P, Gottlieb L (2014) The social determinants of health: it's time to consider the causes of the causes. Pub Health Rep 129: 19-31.

30. Fordal O, Lygre H (2015) Management of periodontal disease in patient using calcium channel blocker - gingival overgrowth, prescribed medications, treatment responses and added treatment costs. J Clin Periodontol 42: 640-6.
31. Sreebny LM, Schwartz SS (1997) A reference guide to drugs and dry mouth-2nd edition. Gerontol 14: 33-47.

32. Guggenheimer J, Moore P (2003) Xerostomia Etiology, recognition and treatment. J Am Dental Associa 134: 61-69.

33. American Dental Association (2019) Action for dental health.

34. Institute of Medicine (IOM) and National Research Council (2011) Improving Access to Oral Health Care for vulnerable and underserved populations.

35. Bloom B, Simile CM, Adams PF, Cohen RA (2012) Oral health status and access to oral health care for U.S. adults aged 18-64: National Health Interview Survey, 2008. National Center for Health Statistics. Vital and Health Statistics 10: 1-22.

36. U.S. Department of Health and Human Services and Center for Disease Control and Prevention Oral health program (2013) Strategic planning for 2011-2014.

37. U.S. Department of Health and Human Services Oral Health Strategic Framework (2016) 2014-2017. Pub Health Rep 131: 242-257.

38. Palmer CA, Gilbert JA (2012) Position of the Academy of Nutrition and Dietetics: the impact of fluoride on health. J Acad Nutrit Dietet 112: 1443-1453.

39. Gonsalves W, Wrightson WS, Henry R (2008) Common oral conditions in older persons. Am Family Physician 78: 845-852.

40. Barber LR, Wilkins EM (2002) Evidence-based prevention, management and monitoring of dental caries, J Dental Hygiene 76: 270-275.

41. Marsh PD (2004) Dental plaque as a microbial biofilm. Caries Res 38: 204-211.

42. Marsh PD (2006) Dental plaque as a biofilm and a microbial community-implications for health and disease. BMC Oral Health 6: s14.

43. Thomson, W (2004) Dental Caries experience in older people over time: what can the large cohort studies tells us? Brit Dental J 96; 89-92.

44. American Dental Association (2019) MouthHealthy website. Oral Health.

45. American Dental Association (2019) MouthHealthy website.

46. American Academy of Periodontology (2019) Types of gum disease.

47. American Academy of Periodontology (2019) Gum Disease Risk factors.

48. American Academy of Periodontology (2019) Gum disease and women.

49. American Dental Association (2019) MouthHealthy website. Gum disease.

50. Amaral CD, Vettore MV, Leao A (2009) The Relationship of alcohol dependence and alcohol consumption with periodontitis: a systemic review. J Dentist 37: 643-651.

51. John CN, Stephen LX, Africa CW (2013) Is human immunodeficiency virus (HIV) stage an independent risk factor for altering the periodontal status of HIV-positive patients? A South African study. BMC Oral Health 13: 69.

52. American Academy of Periodontology (2019) Patient resources.

53. American Dental Association (2019) Mouth Healthy website. Brushing your teeth.

54. Ameet MM, Avneesh HT, Babita RP, Pramod PM (2013) The relationship between periodontitis and systemic diseases - hype or hope? J Clini Diagnostic Res 7: 758-62.

55. Barnett ML (2006) The oral-systemic disease connection. J Am Dental Associa 137: 5S-6S. 
56. Scannapieco, F. Pueumonia in nonambulatory patients (2006) The role of oral bacteria and oral hygiene. J Am Dental Associa 137: 21S-25S

57. Li L, Ai Z, Li L, Zheng X, Jie L et al. (2015) Can routine oral care with antiseptics prevent ventilator-associated pneumonia in patients receiving mechanical ventilation? An update metaanalysis from 17 randomized controlled trials. Int J Clini Exp Med 8: 1645-1657.

58. Barnett ML, Hyman JL (2006) Challenges in interpreting study results. J Am Dental Associa 137: 32S-36S

59. Center for Disease Control and Prevention (2013) Periodontal disease.

60. American Academy of Periodontology (2019) Gum disease risk factors.

61. Pretty IA, Elllwood RP, Lo EC (2014) The Seatle Care Pathway for Securing oral health in older patients. Gerontol 1: 77-87.

62. Park H, Suk S, Cheong J, Lee H, Chang H, et al. (2013) Tooth loss may predict poor cognitive function in community-dwelling adults without dementia or stroke: The PRESENT project. J Korean Med Sci 28: 1518-1521.

63. Kaye EK, Valencia A, Baba N, Spiro A, Dietrich T, et al. (2010) Tooth loss and periodontal disease predict poor cognitive function in older men. J Am Geriatr Soc 58: 713-718.

64. Griffin SO, Jones JA, Brunson D, Griffin PM, Bailey WD, et al. (2012) Burden of oral disease among older adults and implications for public health priorities. Am J Pub Health 102: 411-418.

65. Federal Interagency Forum on Aging-Related Statistics (2016) Older Americans, Key indicators of wellbeing.

66. Eke PI, Dye BA, Wei L (2015) Update on prevalence of periodontitis in adults in the United States. NHANES 20092012. J Periodontol 86: 611-622.

67. Lee JY, Divaris K, Baker AD, Rozier RG, Vann WF Jr, et al. (2012) The relationship of oral health literacy and self-efficacy with oral health status and dental neglect. Am J Pub Health. 102: 923-929.

68. Hewitt M (2013) Oral health literacy workshop summary. Institute of Medicine of The National Academies.

69. National Institute of Dental and Craniofacial Research (2005) National Institutes of Health, US Public Health Service, US Dept of Health and Human Services. The invisible barrier: literacy and its relationship with oral health. J Pub Health Dentist 65: 174-182.

70. Institute of Medicine of The National Academies (2011) Advancing oral health in America.

71. Coleman P, Watson NM (2011) Oral care provided by certified nursing assistants in nursing homes. J Am Geriatr Soc 54: 138143.

72. Stein PS, Aalboe JA, Svage MW, Scott AM (2014) Strategies for communicating with older dental patients. Am Dental Associa 145: 159-164.

73. Taylor DW, Loesche WJ, Terpenning MS (2000) Impact of oral diseases on systematic health in the elderly: diabetes mellitus and aspiration pneumonia. J Pub Health Dentistry 60: 313-20.

74. Taylor GW, Manz MC, Borgnakke WS (2004) Diabetes, periodontal diseases, dental caries, and tooth loss: a review of the literature. Compendium of Continuing Edu Dentist 25: 179-84.

75. Huang DL, Chan KC, Young BA (2013) Poor oral health and quality of life in older U.S. adults with diabetes mellitus. J Am Geriatr Soc 61: 1782-8.
76. U.S. National Library of Medicine (2019) MedlinePlus. Thrush - children and adults.

77. CMS.gov (2018) Center for Medicare \& Medicaid Services. Medicare program: general information.

78. CMS.gov (2013) Center for Medicare \& Medicaid Services. Medicare dental coverage.

79. Kaiser Family Foundation Medicare Policy (2012) Oral health and medicare beneficiaries: coverage, out-of-pocket spending, and unmet need. Issue Brief.

80. Center for Disease Control and Prevention (2019) Oral health of older Americans.

81. Vargas CM, Kramarow EA, Yellowitz JA (2001) Oral health of older Americans. Aging Trends 1-8. Hyattsville, MD: National Center for Health Statistics.

82. Center For Medicare Advocacy (2013) New Report: Expanded dental coverage needed to confront health crisis.

83. Manski RJ, Brown E (2007) Dental use, expenses, private dental coverage, and changes, 1996 and 2004. Rockville, MD: Agency for Healthcare Research and Quality.

84. Oral Health America (2013) State of decay. Are older Americans coming of age without oral healthcare?

85. Center for Disease Control (2016) Disparities in oral health

86. Center for Disease Control. C and Prevention (2019)n Community water fluoridation.

87. National Institute of Dental and Craniofacial Research (2019) Tooth decay.

88. NHS. Tooth decay. April 2019.

89. Hellwig E, Lussi A (2014) Oral hygiene products, medications and drugs - hidden aetiological factors for dental erosion. Monographs in Oral Sci 25: 155-62.

90. National Institute of Dental and Craniofacial Research (2018) Dry mouth.

91. Salinas T (2017) Dry mouth treatment: Tips for controlling dry mouth.

92. U.S. Department of Health and Human Services (2003) National Call to Action to Promote Oral Health. Rockville, MD: U.S. Department of Health and Human Services, Public Health Service, National Institutes of Health, National Institute of Dental and Craniofacial Res, NIH Publication No. 03-5303.

93. Dye BA, Tan S, Smith V (2007) Trends in oral health status: United States, 1988-1994 and 1999-2004, Vital Health Statistics 1-92.

94. Center for Disease Control and Prevention (2018) Alcohol and public health. Fact sheets - alcohol use and health.

95. Mandel L (2005) Dental erosion due to wine consumption. J Am Dental Associa 136: 71-75.

96. Brand HS, Tjoe GM, Veerman EC (2009) The effect of saliva on the erosive potential of three different wines. Aus Dental J 5493: 228-232.

97. Menarte P, Manso C, Souza D, Frias-Bulhosa J, Gago S, et al. (2009) Dental erosion in alcoholic patients under addiction rehabilitation therapy. Oral Med Pathology 14: e377-84.

98. Willershausen B, Callaway A, Azrak,B, Klob C, SchulzDobrick B, et al. (2009) Prolonged in vitro exposure to white wines enhances the erosive damage on human permanent teeth compared with red wines. Nutrition Sci 29: 558-567.

99. Noel-Miller C (2012) Medicare beneficiaries' out-of-pocket spending for health care. Insight on the Issues 65: 1-8. 
100. Vujicic M, Buchmueller T, Klein R (2016) Dental Care Presents The Highest Level Of Financial Barriers, Compared To Other Types Of Health Care Services. Health Affairs (Millwood) 35: 2176-2182.

101. Vujicic M (2018) Our dental care system is stuck: And here is what to do about it. J Am Dental Associa 149: 167-169.

102. Watts A, Addy M (2001) Tooth discolouration and staining: a review of the literature. Brit Dental J 190: 309-316.

103. Sulieman M (2005) An overview of tooth discoloration: extrinsic, intrinsic and internalized stains. Dental Update, 32: 463-4, 466-8, 471.

104. Darby ML, Walsh MM (2010) Oral hygiene assessment: soft and hard deposits. In: Darby, ML \& Walsh MM. Dental Hygiene Theory and Pract 274-276.

105. Krastl G, Allgayer N (2013) Tooth discoloration induced by endodonticmaterials: aliteraturereview.DentalTraumatol29:2-7.

106. American Dental Association - MouthHealthy website. Mouth sores.

107. Epstein JB, Polsky B (2009) Oropharyngeal candidiasis: a review of its clinical spectrum and current therapies. Clin Therapeutics 20: 40-57.

108. Centers for Disease Control and Prevention (2019) Candidiasis infections of the mouth, throat, and esophagus.

109. Gendreau L, Loewy ZG (2011) Epidemiology and etiology of denture stomatitis. J Prosthodontics 20: 251-260.

110. American Academy of Periodontology (2019) Gum disease symptoms.

111. American Academy of Periodontology (2019) Preventing periodontal disease.

112. MacDonald DE (2006) Principles of geriatric dentistry and their application to the older adult with a physical disability. Clini Geriatr Med 22: 413-434.

113. U.S National Library of Medicine (2019) Periodontitis. MedlinePlus.

114. U.S National Library of Medicine (2019) Bleeding Gums. MedlinePlus.

115. Scully C, Porter S (2000) ABC of Oral Health Swellings and red, white, and pigmented lesions. Brit Med J 321: 225-228.

116. Hiremath VK, Hussein A, Mishra N (2011) Prevelance of torus palatinus and torus mandibularis among Malay population. J Int Soc Preven Commu Dentistry 1: 60-64.

117. Meskin LH (1996) A Breath of Fresh Air. J Am Dental Associa 127: $1282-1284$.

118. Scully C, Greenman J (2012) Halitology (breath odour: aetiopathogenesis and management). Oral Diseases 18:333-345.

119. Liu XN, Shibnada K, Chen XC, Zhang BX, Yaegtak, K, et al. (2000) Oral malador-related parameters in the Chinese general population. J Clini Periodontol 33: 31-36.

120. Outhouse TL, Al-Alawi R, Fedorowicz Z, Keenan JV (2000) Tongue scraping for treating halitosis. Cochrane Database Systematic Rev CD005519.

121. Zalewska A, Zatonski M, Jablonka-Strom A, Paradowska A, Kawala B, et al. (2012) Halitosis - a common medical and social problem. A review on pathology, diagnosis and treatment. Acta Gastroenterol Belgica 75: 300-309.

122. American Dental Association Council on Scientific Affairs (2003) Oral malodor. J Am Dental Associa134; 209-214.

123. Porter SR, Scully C (2006) Oral malodour (halitosis). Brit Dental J 333: 632-635.
124. Jablonski RA, Therrien B, Klanowski A (2011) No More Fighting and Biting During Mouth Care: Applying the Theoretical Constructs of Threat Perception to Clinical Practice. Res Theory Nurs Pract 25: 163-175.

125. Jablonski RA, Swecker T, Munro C, Grap MJ, Ligon M, et al. (2009) Measuring the Oral Health of Nursing Home Elders. Clinil Nurs Res 18: 200-217.

126. American Dental Association of Science. Oral cleansers (2003) J Am Dental Associa 134: 108-109.

127. Grap MJ, Munro CL, Ashtiani B, Bryant S (2003) Oral care interventions in critical care: frequency and documentation. Am J Critical Care 12: 113-118.

128. Product of Kimberly-Clark Corporation

129. Lagacé M, Tanguay A, Lavallee M, Laplante J, Robichaud S, et al. (2012) The silent impact of ageist communication in long term care facilities: elders' perspectives on quality of life and coping strategies. J Aging Stud 26: 335-342.

130. Williams KN, Herman R, Gajewski B, Wilson K (2009) Elderspeak communication: impact on dementia care. American J Alzheimer's Disease and Other Dementias 24: 11-20.

131. Persson GR (2006) What has ageing to do with periodontal health and disease? Int Dental J 56: 240-249.

132. Petersen PE, Bourgeois D, Ogawa H, Estupinan-Day S, Ndiaye $\mathrm{C}$, et al. (2005) The global burden of oral diseases and risk to oral health. Bulletin of the World Health Organization 83: 661669.

133. Liu Y, Li Z, Walker MP (2014) Social disparities in dentition status among American adults. Int Dental J 64: 52-57.

134. Savoca MR, Arcury TA, Leng X, Cheng H (2010) Severe tooth in older adults as a key indicator of compromised diet quality. Pub Health Nutrit 13: 466-474.

135. Savoca MR, Arcury TA, Leng X, Cheng H (2010) Food Avoidance and Food Modification Practices due to Oral Health Problems Linked to the Dietary Quality of Older Adults. J Am Geriatr Soc 58: 1225-1232.

136. Quandt SA, Savoca MR, Leng X, Chen H (2011) Dry mouth and dietary quality among older adults in North Carolina. J Am Geriatr Soc 59: 439-445.

137. American Dental Association. Action for dental health. Access to care.

138. National Institute of Dental and Craniofacial Research (NIDCR) (2018) Treatment Needs in Seniors (Age 65 and Over).

139. U.S. Department of Health and Human Services (2014) Health Resources and Services Administration (HRSA). Integration of oral health and primary car practice. 